\title{
Generic primal-dual solvability in continuous linear semi-infinite programming
}

\author{
M.A. GOBERNA $\dagger^{*}$, M.I. TODOROV** $\ddagger$ \\ $\dagger$ Department of Statistics and Operations Research, University of Alicante, \\ 03071 Alicante, Spain \\ ‡ Operations Research Center, Miguel Hernández University of Elche, \\ 03202 Elche (Alicante), Spain
}

(Received Day Month 2006; in final form Day Month 2007)

In this paper we consider the space of all the linear semi-infinite programming (LSIP) problems with a given infinite compact Hausdorff index set, a given number of variables, and continuous coefficients, endowed with the topology of the uniform convergence. These problems are classified as inconsistent, solvable with bounded optimal set, bounded (i.e., finite valued) but either unsolvable or having an unbounded optimal set, and unbounded (i.e., with infinite optimal value), giving rise to the socalled refined primal partition of the space of problems. The mentioned LSIP problems can be also classified with a similar criterion applied to the corresponding Haar's dual problems, which provides the refined dual partition of the space of problems. We characterize the interior of the elements of the refined primal and dual partitions as well as the interior of the intersections of the elements of both partitions (the so-called refined primal-dual partition). These characterizations allow to prove that most (primal or dual) bounded problems have simultaneously primal and dual non-empty bounded optimal set. Consequently, most bounded continuous LSIP problems are primal and dual solvable.

Keywords: linear programming; linear semi-infinite programming; stability

AMS Subject Classifications 2000: 90C34, 90C31, 49K40

*Corresponding author. E-mail: mgoberna@ua.es. Research supported by DGES and FEDER, Grant MTM2005-08572-C03-01.

**E-mail: maxim.todorov@udlap.mx. Research partially supported by CONACyT of MX. Grant 44003 


\section{Introduction}

Throughout this paper we consider given an infinite compact Hausdorff topological space $T$ and a natural number $n \geq 2$. We associate with each triple $\pi=(a, b, c) \in \Pi:=\mathcal{C}(T)^{n} \times \mathcal{C}(T) \times \mathbb{R}^{n}$ a (continuous) linear semi-infinite programming (LSIP) problem

$$
\begin{aligned}
& P \text { : Min } c^{\prime} x \\
& \text { s.t. } \quad a_{t}^{\prime} x \geq b_{t}, \quad t \in T,
\end{aligned}
$$

and its corresponding Haar's dual problem

$$
\begin{aligned}
D: \operatorname{Max} & \sum_{t \in T} \lambda_{t} b_{t} \\
\text { s.t. } & \sum_{t \varepsilon T} \lambda_{t} a_{t}=c, \\
& \lambda_{t} \geq 0, t \in T,
\end{aligned}
$$

where $\lambda: T \mapsto \mathbb{R}$ satisfies that $\lambda_{t}=0$ for all $t \in T$ except maybe for a finite number of indices. The decision space of $D$ is denoted by $\mathbb{R}^{(T)}$ (the linear space of generalized finite sequences) and its positive cone by $\mathbb{R}_{+}^{(T)}$. The feasible (optimal) sets of $D$ and $P$ are denoted by $\Lambda$ and $F\left(\Lambda^{*}\right.$ and $F^{*}$, respectively)

Among the well-known applications of continuous LSIP let us mention that problems like $P$ arise in functional approximation, separation, pollution control, finance, Bayesian statistics and the design of telecommunications networks, whereas problems like $D$ have been used in robust Bayesian analysis and optimization under uncertainty. These applications are described in [8, Chapters 1-2], [7], and the references therein. Recall that, in contrast with ordinary linear programming, in continuous LSIP the bounded (primal or dual) problems are not necessarily solvable (i.e., there exist finite-valued problems with no optimal solution).

We consider $\mathbb{R}^{(T)}$ equipped with one of the norms $l_{\infty}$ or $l_{1}$. The space of parameters $\Pi$ can be interpreted as the set of triples (or dual pairs of problems) obtained by means of arbitrary perturbations performed on a fixed triple $\pi=(a, b, c)$, provided such perturbations preserve the structure of $\pi$, i.e., the number of variables, the index set and the continuity of the coefficient functions. We say that a certain desirable property holds generically in a certain set $\Delta \subset \Pi$ when this property holds for all the elements of an open dense subset of $\Delta$ (for the topology of the uniform convergence on $\Pi$ defined in Section 2). Typical desirable properties of convex optimization problems are uniqueness of the optimal solution (strong uniqueness in the case of linear objective func- 
tion) or at least non-emptyness and boundedness of the optimal set. This last property plays a crucial role in convergence analysis (see, e.g., [1, Chapter 3]). We denote by $\Pi_{C}^{P}, \Pi_{I C}^{P}, \Pi_{B}^{P}$, and $\Pi_{U B}^{P}\left(\Pi_{C}^{D}, \Pi_{I C}^{D}, \Pi_{B}^{D}\right.$, and $\left.\Pi_{U B}^{D}\right)$ the classes of parameters providing primal (dual) consistent, inconsistent, bounded (i.e., with finite optimal value), and unbounded problems, respectively. We are concerned with those parameters which are stable for the corresponding property in the sense that sufficiently small perturbations of the parameter preserve its membership. The interior of $\Pi_{C}^{P}, \Pi_{I C}^{P}, \Pi_{C}^{D}$, and $\Pi_{I C}^{D}$ where characterized in [9] and [10], whereas [3], [12] and [13] have characterized the interior of the elements of the primal partition (of $\Pi$ ), $\left\{\Pi_{I C}^{P}, \Pi_{B}^{P}, \Pi_{U B}^{P}\right\}$, the dual partition, $\left\{\Pi_{I C}^{D}, \Pi_{B}^{D}, \Pi_{U B}^{D}\right\}$, and the primal-dual partition, which is formed by the nonempty pairwise intersections of the primal and the dual partitions, identifying those elements of the mentioned partitions which have non-empty interior and showing that each non-empty interior is a dense subsets in the corresponding class. Concerning the primal-dual partition, let us observe that its elements are formed by those parameters sharing the same duality state (see [2], [17], [21] and [18] for duality states in mathematical programming, the last one dealing with LSIP and semi-definite programming).

In this paper we extend the previous analysis to the refined partitions which result of classifying any bounded problem as having a non-empty bounded optimal set or not satisfying this desirable property (in which case the bounded problem can be either unsolvable or solvable with an unbounded optimal set). This criterion splits $\Pi_{B}^{P}$ into the sets $\Pi_{S}^{P}$ and $\Pi_{N}^{P}$. Similarly, from the dual perspective, we decompose $\Pi_{B}^{D}$ into the sets of parameters providing dual problems with bounded optimal sets, $\Pi_{S}^{D}$, and its complement relative to $\Pi_{B}^{D}$, denoted by and $\Pi_{N}^{D}$. Thus, we obtain the refined primal partition, $\left\{\Pi_{I C}^{P}, \Pi_{S}^{P}, \Pi_{N}^{P}, \Pi_{U B}^{P}\right\}$, the refined dual partition, $\left\{\Pi_{I C}^{D}, \Pi_{S}^{D}, \Pi_{N}^{D}, \Pi_{U B}^{D}\right\}$, and the refined primal-dual partition, which is formed by the non-empty pairwise intersections of the last two partitions.

In [22] was shown that $\Pi_{B}^{P}$ contains a $G_{\delta}$ (intersection of a countable family of open sets) dense subset of parameters with strongly unique primal optimal solution. In [6] the $G_{\delta}$-set was replaced by an open set, i.e., it was shown that the strong uniqueness holds generically in $\Pi_{B}^{P}$. The generic property analyzed in this paper is the primal-dual solvability (i.e., of both associated problems), with bounded optimal sets. This property is weaker than the previous one in the sense that it does not imply the primal strongly uniqueness of the approximating problems, but it is also stronger in the sense that the approximating problems and their dual problems are simultaneously solvable with bounded optimal sets.

The new results appear in Sections 3 and 4. In Section 3 we identify the elements of the refined primal-dual partition, characterizing their respective interiors whereas in Section 4 we provide similar results for the elements of the 
refined primal and dual partitions. From the characterizations of the interiors of these sets we prove, in both sections, that primal-dual solvability with bounded optimal set is a generic property in $\Pi_{B}^{P} \cup \Pi_{B}^{D}$ (the class of parameters for which at least one of the two associated problems is bounded). A suitable counterexample shows that the last statement is not true in general LSIP.

\section{Preliminaries}

The null-vector in $\mathbb{R}^{p}$ is denoted by $0_{p}$ and the $j$ th element of the canonical basis by $e_{j}$. Given a non-empty set $X \subset \mathbb{R}^{p}$, conv $X$ and cone $X$ denote the convex hull of $X$ and the conical convex hull of $X \cup\left\{0_{p}\right\}$, respectively (so that cone $\emptyset=\left\{0_{p}\right\}$ ). If $X$ is convex, $\operatorname{dim} X$ denotes its dimension. From the topological side, if $X$ is a subset of any topological space, $\operatorname{int} X, \operatorname{cl} X, \operatorname{bd} X$, and $\operatorname{acc} X$ represent the interior, the closure, the boundary, and the set of $\omega$ accumulation points of $X$, respectively. Finally, $\lim _{r} x_{r}=x$ (in short $x_{r} \rightarrow x$ ) must be interpreted as $\lim _{r \rightarrow \infty} x_{r}=x$.

The next lemma guarantees the existence of continuous real functions on $T$ satisfying certain conditions. Such elements of $\mathcal{C}(T)$ will allow us to construct ad hoc elements of $\Pi$.

Lemma 2.1 Let $T$ be an infinite compact Hausdorff space and $m \in \mathbb{N}$. Then there exists a point $\bar{t} \in \operatorname{acc} T, m$ subsets of $T, S_{1}, \ldots, S_{m}$, and $m$ functions in $\mathcal{C}(T)$ and values in $[0,1], \varphi_{1}, \ldots, \varphi_{m}$, such that $\varphi_{i}\left(S_{i}\right) \subset \mathbb{R}_{++}$and $\varphi_{i}(\bar{t})=0 \in$ $\operatorname{acc} \varphi_{i}\left(S_{i}\right)$, for all $i=1, \ldots, m$, and $\varphi_{i}\left(S_{j}\right)=\{0\}$ for all $i, j=1, \ldots, m$ such that $i \neq j$.

Proof We use the following consequence of Urisohn's Lemma (already used in the proof of [10, Theorem 6.4(iii)]): there exists a point $\bar{t} \in \operatorname{acc} T$ and a sequence of non-repeated indices $\left\{t_{r}\right\}_{r=1}^{\infty} \subset T$, with $t_{r} \rightarrow \bar{t}$, and a sequence $\left\{f_{r}\right\} \subset \mathcal{C}(T)$, with $f_{r}: T \rightarrow[0,1]$, such that $f_{r}\left(t_{r}\right)=1$ and $f_{r}\left(t_{k}\right)=0$ for all $k \neq r$.

It is easy to see that the sets $S_{i}:=\left\{t_{m r+i}, r=1,2, \ldots\right\}$ and the functions $\varphi_{i}:=\sum_{r=1}^{\infty} 2^{-(m r+i)} f_{m r+i}, i=1, \ldots, m$, satisfy all the requirements.

We denote by $v^{P}(\pi)\left(v^{D}(\pi)\right)$ the optimal value of $P(D)$, defining as usual $v^{P}(\pi)=+\infty\left(v^{D}(\pi)=-\infty\right.$, respectively) when the corresponding problem is inconsistent. Since $P$ and $D$ can be either inconsistent (IC) or bounded (B) or unbounded (UB), we get at most nine possible duality states, which are reduced to six by the weak duality theorem: $v^{D}(\pi) \leq v^{P}(\pi)$. The first row in Table 1 contains the elements of the primal partition, the first column are the elements of the dual partition, and the sets in the remaining cells, $\Pi_{1}, \ldots, \Pi_{6}$, are 
the non-empty intersections of the corresponding entries (i.e., $\Pi_{1}=\Pi_{B}^{P} \cap \Pi_{B}^{D}$, etc.), so that they are the elements of the primal-dual partition.

\begin{tabular}{|c||c|c|c|}
\cline { 2 - 4 } \multicolumn{1}{c||}{} & $\Pi_{I C}^{P}$ & $\Pi_{B}^{P}$ & $\Pi_{U B}^{P}$ \\
\hline \hline$\Pi_{I C}^{D}$ & $\Pi_{4}$ & $\Pi_{5}$ & $\Pi_{2}$ \\
\hline$\Pi_{B}^{D}$ & $\Pi_{6}$ & $\Pi_{1}$ & \\
\hline$\Pi_{U B}^{D}$ & $\Pi_{3}$ & & \\
\hline
\end{tabular}

Table 1

As most of the works on perturbation theory in continuous LSIP (e.g., the classical paper [20]), we consider $\Pi$ equipped with the metric of the uniform convergence, i.e., given $\pi^{i}=\left(a^{i}, b^{i}, c^{i}\right) \in \Pi, i=1,2$, the distance between $\pi^{1}$ and $\pi^{2}$ is

$$
d\left(\pi^{1}, \pi^{2}\right)=\max \left\{\left\|c^{1}-c^{2}\right\|_{\infty}, \max _{t \in T}\left\|\left(\begin{array}{c}
a_{t}^{1} \\
b_{t}^{1}
\end{array}\right)-\left(\begin{array}{c}
a_{t}^{2} \\
b_{t}^{2}
\end{array}\right)\right\|_{\infty}\right\}
$$

From now on, the same subscripts or superscripts that distinguish the elements of $\Pi$ will distinguish also their corresponding objects: $\pi^{r}=\left(a^{r}, b^{r}, c^{r}\right)$, $D_{r}, P_{r}, \Lambda_{r}, F_{r}$, and so on. Thus, according to Farkas lemma, if $\pi^{1}, \pi^{2} \in \Pi_{C}^{P}$, then $F_{1}=F_{2}$ if and only if $\mathrm{cl} K_{1}=\operatorname{cl} K_{2}$.

We will show that some results in this paper are not valid for general LSIP, where $T$ is an arbitrary infinite set (not even a topological space) and the functions in the triple $\pi=(a, b, c)$ are also arbitrary. To do that we need some additional notation. In this general setting the space of parameters is $\Theta:=\left(\mathbb{R}^{n}\right)^{T} \times \mathbb{R}^{T} \times \mathbb{R}^{n}$ equipped with the pseudometric which generates in $\Theta$ the topology of the uniform convergence (replacing "max" with "sup" in (1)) introduced in [14]. We denote the relevant subsets of $\Theta$ with the same subscripts and upperscripts as for the corresponding subsets of $\Pi$ (i.e., $\Theta_{C}^{P}$ stands for the parameters with consistent primal problem, etc.).

Next we recall some concepts and basic results we will use on general LSIP (all the proofs and references can be found in [8]). We associate with $\pi=$ $(a, b, c)$ its first and second moment cones of $\pi, M:=$ cone $\left\{a_{t}, t \in T\right\}$ and $N:=$ cone $\left\{\left(a_{t}, b_{t}\right), t \in T\right\}$, and its characteristic cone, $K:=N+\mathbb{R}_{+}\left\{\left(0_{n},-1\right)\right\}$. Moreover, if $P$ is consistent and $N$ is closed, then $K$ is closed too. If $D$ is consistent and $K$ is closed, then $\sup \{\alpha \in \mathbb{R} \mid(c, \alpha) \in K\}$ is attained and, so, $D$ is solvable. The existence theorem establishes that $P$ is consistent if and only if $\left(0_{n}, 1\right) \notin \mathrm{cl} K$. In such a case, the non-homogeneous Farkas lemma establishes that the inequality $c^{\prime} x \geq d$ holds for all $x \in F$ if and only if $(c, d) \in \operatorname{cl} K$.

The above results are also valid in continuous LSIP, where the Slater con- 
straint qualification plays a crucial role. Recall that $\pi=(a, b, c)$ satisfies the Slater condition if there exists $\bar{x} \in \mathbb{R}^{n}$ such that $a_{t}^{\prime} \bar{x}>b_{t}$ for all $t \in T$. The Slater condition holds if and only if $0_{n+1} \notin \operatorname{conv}\left\{\left(a_{t}, b_{t}\right), t \in T\right\}$. If $\pi$ satisfies the Slater condition, then $N$ is closed.

We use the following characterizations of $\Pi_{S}^{P}$ and $\Pi_{S}^{D}$ (see [8, Corollary 9.3.1 and Theorem 9.8]).

LEMMA 2.2 (i) $\pi \in \Pi_{S}^{P}$ if and only if $\left(0_{n}, 1\right) \notin \operatorname{cl} K$ and $c \in \operatorname{int} M$.

(ii) $\pi \in \Pi_{S}^{D}$ if and only if $c \in M$ and Slater condition holds.

The next example shows that Lemma 2.2 is not true in general LSIP

Example 2.3 Let $T=\mathbb{N}^{2}, n=2$ and $\pi=(a, b, c) \in \Theta$ be such that $a$ is the identity map on $T, b=-1$ and $c=(1,1)$. It is easy to see that $K_{1}=$ $\left(\mathbb{R}_{++}^{2} \times \mathbb{R}_{-}\right) \cup\left\{0_{2}\right\}, c^{1} \in \operatorname{int} M_{1}=\mathbb{R}_{++}^{2}$, the Slater condition holds (take $\left.\bar{x}=0_{2}\right), F_{1}^{*}=\left\{0_{2}\right\}$, and $\sup \left\{\alpha \in \mathbb{R} \mid\left(c^{1}, \alpha\right) \in K_{1}\right\}=0$ is not attained for all $\pi^{1}=\left(a^{1}, b^{1}, c^{1}\right) \in \Theta$ such that $d\left(\pi^{1}, \pi\right)<\frac{1}{2}$. Thus we have an open subset of $\Pi_{S}^{P}$ where statement (ii) in Lemma 2.2 fails.

Let us interpret the topological interior of some elements of the partitions considered in this paper. A LSIP problem is called ill-posed in the feasibility sense if arbitrarily small perturbations provide both consistent and inconsistent problems (the distance to ill-posedness in $\Theta$ has been characterized in [3]). Consequently, the sets int $\Pi_{C}^{P}$ and $\operatorname{int} \Pi_{I C}^{P}\left(\operatorname{int} \Pi_{C}^{D}\right.$ and $\left.\operatorname{int} \Pi_{I C}^{D}\right)$ can be seen as the set of primal (dual) stable problems (these interiors have been characterized in [9], [8] and [10]). On the other hand, [19] defines a conic programming problem to be ill-posed (in primal-dual feasibility sense) when it lays on the boundary of the set of parameters providing consistent primal and dual problems. This class of primal-dual ill-posed parameters is, in our setting, bd $\Pi_{1}$. The following lemma summarizes results on the primal-dual partition which appeared in [22] (where int $\Pi_{1}$ was characterized), [3], [12, Section 4] (taking into account that $N$ can be replaced with $K$ in all the characterizations) and [13].

LEMma 2.4 The elements of the primal-dual partition are neither open nor closed and satisfy the following statements:

(i) $\pi \in \Pi_{1}$ if and only if $\left(0_{n}, 1\right) \notin \mathrm{cl} K$ and $c \in M$. In particular, $\pi \in \operatorname{int} \Pi_{1}$ if and only if Slater condition holds and $c \in \operatorname{int} M$. Moreover, int $\Pi_{1}$ is dense in $\Pi_{1}$.

(ii) $\pi \in \Pi_{2}$ if and only if $\left(0_{n}, 1\right) \notin \operatorname{cl} K, c \notin M$ and $(\{c\} \times \mathbb{R}) \cap \operatorname{cl} K=\emptyset$. In particular, $\pi \in \operatorname{int} \Pi_{2}$ if and only if there exists $y \in \mathbb{R}^{n}$ such that $c^{\prime} y<0$ and $a_{t}^{\prime} y>0$ for all $t \in T$. Moreover, int $\Pi_{2}$ is dense in $\Pi_{2}$.

(iii) $\pi \in \Pi_{3}$ if and only if $\left(0_{n}, 1\right) \in \mathrm{cl} K, c \in M$ and $\{c\} \times \mathbb{R} \subset K$. In particular, $\pi \in \operatorname{int} \Pi_{3}$ if and only if $\left(0_{n}, 1\right) \in \operatorname{int} K$. Moreover, int $\Pi_{3}$ is dense in $\Pi_{3}$. 
(iv) $\pi \in \Pi_{4}$ if and only if $\left(0_{n}, 1\right) \in \operatorname{cl} K$ and $c \notin M$. Moreover, int $\Pi_{4}=\emptyset$.

(v) $\pi \in \Pi_{5}$ if and only if $\left(0_{n}, 1\right) \notin \operatorname{cl} K, c \notin M$ and $(\{c\} \times \mathbb{R}) \cap \operatorname{cl} K \neq \emptyset$. Moreover, int $\Pi_{5}=\emptyset$.

(vi) $\pi \in \Pi_{6}$ if and only if $\left(0_{n}, 1\right) \in \mathrm{cl} K, c \in M$ and $\{c\} \times \mathbb{R} \nsubseteq \subseteq K$. Moreover, int $\Pi_{6}=\emptyset$.

(vii) $\Pi_{i} \subset \operatorname{clint} \Pi_{j}$ for all $(i, j) \neq(4,1), i=4,5,6, j=1,2,3$.

Let us observe that the recent paper [5] provides characterizations of the interior, the boundary and the exterior of the sets $\Theta_{C}^{D}$ and $\Theta_{1}$ in general LSIP. Obviously, these characterizations become sufficient conditions in the context of continuous LSIP. In general LSIP, int $\Theta_{B}^{P}$ coincides with the interior of the class of those $\pi \in \Theta$ such that the corresponding primal problem is solvable ( $[4$, Theorem 1]).

\section{The refined primal-dual partition}

Table 2 is the counterpart of Table 1 for the refined partitions, although some of the crossed intersections could be empty:

\begin{tabular}{|c||c|c|c|c|}
\cline { 2 - 5 } \multicolumn{1}{c|}{} & $\Pi_{I C}^{P}$ & $\Pi_{S}^{P}$ & $\Pi_{N}^{P}$ & $\Pi_{U B}^{P}$ \\
\hline \hline$\Pi_{I C}^{D}$ & $\Pi_{4}$ & & $\Pi_{5}$ & $\Pi_{2}$ \\
\hline$\Pi_{S}^{D}$ & & $\Pi_{1}^{1}$ & $\Pi_{1}^{3}$ & \\
\hline$\Pi_{N}^{D}$ & $\Pi_{6}$ & $\Pi_{1}^{2}$ & $\Pi_{1}^{4}$ & \\
\hline$\Pi_{U B}^{D}$ & $\Pi_{3}$ & & & \\
\hline
\end{tabular}

Table 2 $\emptyset$.

We must justify the empty cells in Table 2 , i.e., that $\Pi_{S}^{P} \cap \Pi_{I C}^{D}=\Pi_{I C}^{P} \cap \Pi_{S}^{D}=$

First, if $\pi \in \Pi_{S}^{P} \cap \Pi_{I C}^{D}$, then $c \in \operatorname{int} M$ and $c \notin M$ (contradiction). Thus $\Pi_{S}^{P} \cap \Pi_{I C}^{D}=\emptyset$ and, so, $\Pi_{N}^{P} \cap \Pi_{I C}^{D}=\Pi_{5}$.

Second, if $\pi \in \Pi_{I C}^{P} \cap \Pi_{S}^{D}$, then $\pi$ is primal inconsistent and satisfies Slater condition (contradiction). Thus $\Pi_{I C}^{P} \cap \Pi_{S}^{D}=\emptyset$ and $\Pi_{I C}^{P} \cap \Pi_{N}^{D}=\Pi_{6}$.

The next result shows that all the intersections in Table 2 are non-empty $\left(\Pi_{i} \neq \emptyset, i=2, \ldots 6\right.$, was shown in $\left.[12]\right)$.

Theorem $3.1 \Pi_{1}^{j} \neq \emptyset, j=1, . ., 4$.

Proof The basic tool of the proof is Lemma 2.1. 
$\underline{\Pi_{1}^{1} \neq \emptyset}$ : Let $\bar{t} \in T, S_{i}^{k} \subset T$ and $\varphi_{i}^{k} \in \mathcal{C}(T), i=1, \ldots, n, k=1,2$, such that

$$
\varphi_{i}^{k}(\bar{t})=0 \in \operatorname{acc} \varphi_{i}^{k}\left(S_{i}^{k}\right) \subset \mathbb{R}_{++},
$$

for all $i$ and $k$, and $\varphi_{i}^{k}\left(S_{j}^{l}\right)=\{0\}$ if $(i, k) \neq(j, l)$. Consider $\pi=(a, b, c)$ such that $a:=\left(\varphi_{1}^{1}-\varphi_{1}^{2}, \ldots, \varphi_{n}^{1}-\varphi_{n}^{2}\right), b:=-1$ and $c \in \mathbb{R}^{n}$ arbitrary. Given $i \in\{1, \ldots, n\}$, since $a\left(S_{i}^{1}\right)=\left\{0_{i-1}\right\} \times \varphi_{i}^{1}\left(S_{i}^{1}\right) \times\left\{0_{n-i}\right\}$, we have cone $\left\{a_{t}, t \in S_{i}^{1}\right\}=\mathbb{R}_{+} e_{i}$; similarly, cone $\left\{a_{t}, t \in S_{i}^{2}\right\}=-\mathbb{R}_{+} e_{i}$. Then

$$
\sum_{i=1}^{n} \text { cone }\left\{a_{t}, t \in S_{i}^{1}\right\}+\sum_{i=1}^{n} \operatorname{cone}\left\{a_{t}, t \in S_{i}^{2}\right\}=\sum_{i=1}^{n} \mathbb{R}_{+} e_{i}-\sum_{i=1}^{n} \mathbb{R}_{+} e_{i}=\mathbb{R}^{n},
$$

and so $M=\mathbb{R}^{n}$ and $c \in \operatorname{int} M=\mathbb{R}^{n}$. Moreover, $\left\{\left(a_{t}, b_{t}\right), t \in T\right\} \subset \mathbb{R}^{n} \times\{-1\}$, so that $0_{n+1} \notin \operatorname{conv}\left\{\left(a_{t}, b_{t}\right), t \in T\right\}$. Thus $\pi \in \Pi_{1}^{1}$.

$\Pi_{1}^{2} \neq \emptyset$ : Let $\bar{t} \in T, S_{i}^{k} \subset T, \varphi_{i}^{k} \in \mathcal{C}(T), i=1, \ldots, n, k=1,2$, and $a$ be as in the previous proof. Let $b:=-\sum_{i=1}^{n}\left(\varphi_{i}^{1}+\varphi_{i}^{2}\right)^{2}$, and $\pi:=(a, b, c)$, where $c \in \mathbb{R}^{n}$ is taken arbitrarily. We have again $c \in \operatorname{int} M=\mathbb{R}^{n}$. On the other hand, $K \subset$ $\mathbb{R}^{n} \times \mathbb{R}_{-}$(because $\left.b \leq 0\right)$ and $\left(a_{\bar{t}}, b_{\bar{t}}\right)=0_{n+1}$, so that Slater condition fails. Thus $\pi \in \Pi_{1}^{2}$.

$\Pi_{1}^{3} \neq \emptyset$ : Let $\bar{t} \in T, S \subset T$ and $\varphi \in \mathcal{C}(T)$ be such that $\varphi(\bar{t})=0 \in \operatorname{acc} \varphi(S) \subset$ $\mathbb{R}_{++}$. Let $\pi=(a, b, c)$, where $a:=\left(\varphi, 0_{n-1}\right), b:=-1$ and $c:=e_{1}$. Then we have $c \in M=\mathbb{R}_{+} e_{1}$, int $M=\emptyset$ and $\left\{\left(a_{t}, b_{t}\right), t \in T\right\} \subset \mathbb{R}^{n} \times\{-1\}$, so that Slater condition holds. Thus $\pi \in \Pi_{1}^{3}$.

$\Pi_{1}^{4} \neq \emptyset$ : Let $\bar{t}, S, \varphi$ and $a$ be as in the last proof. Let $\pi=(a, b, c)$, where $b \overline{:=-\varphi^{2}}$ and $c:=e_{1}$. We have again $c \in M$ and int $M=\emptyset$. Moreover, $\left(a_{\bar{t}}, b_{\bar{t}}\right)=0_{n+1}$ and $K \subset \mathbb{R}^{n} \times \mathbb{R}_{-}$(because $b \leq 0$ ), so that Slater condition fails and $\left(0_{n}, 1\right) \notin \operatorname{cl} K$. Hence $\pi \in \Pi_{1}^{4}$.

Concerning the parameter $\pi \in \Pi_{1}^{1}$ constructed in the first part of the proof of Theorem 3.1, we cannot assert the uniqueness of the primal and dual optimal solutions. For particular compact Hausdorff spaces $T$, it is possible to give examples where this double uniqueness holds.

Example 3.2 Let $T=\left\{t \in \mathbb{R}^{n} \mid\|t\|=1\right\}$ and $\pi=(a, b, c)$ such that $a$ is the identity mapping on $T, b:=-1$ and $c:=e_{1}$. Then $F=T, F^{*}=\left\{-e_{1}\right\}$, $v^{P}(\pi)=v^{D}(\pi)=-1$ and $\Lambda^{*}=\left\{\lambda^{*}\right\}$, where $\lambda_{t}^{*}=1$ if $t=e_{1}$ and $\lambda_{t}^{*}=0$ otherwise. 
Lemma 2.4 characterizes $\Pi_{1}, \ldots, \Pi_{6}$ and their corresponding interiors, showing that int $\Pi_{i} \neq \emptyset$ is dense in $\Pi_{i}, i=1,2,3$, whereas int $\Pi_{i}=\emptyset, i=4,5,6$. Now we consider the partition of $\Pi_{1}$, i.e., the sets $\Pi_{1}^{j}, j=1, \ldots, 4$.

Theorem 3.3 (i) $\pi \in \Pi_{1}^{1}$ if and only if $c \in \operatorname{int} M$ and the Slater condition holds. Moreover, $\Pi_{1}^{1}$ is an open dense subset of $\Pi_{1}$.

(ii) $\pi \in \Pi_{1}^{2}$ if and only if $\left(0_{n}, 1\right) \notin \mathrm{cl} K, c \in \operatorname{int} M$, and the Slater condition fails. Moreover, $\Pi_{1}^{2}$ is neither closed nor open and int $\Pi_{1}^{2}=\emptyset$.

(iii) $\pi \in \Pi_{1}^{3}$ if and only if Slater condition holds and $c \in M \backslash(\operatorname{int} M)$. Moreover, $\Pi_{1}^{3}$ is neither closed nor open and int $\Pi_{1}^{3}=\emptyset$.

(iv) $\pi \in \Pi_{1}^{4}$ if and only if $\left(0_{n}, 1\right) \notin \operatorname{cl} K, c \in M \backslash$ (int $M$ ), and the Slater condition fails. Moreover, $\Pi_{1}^{4}$ is neither closed nor open and int $\Pi_{1}^{4}=\emptyset$.

Proof The characterizations of the four sets $\Pi_{1}^{j}, j=1, \ldots, 4$, are consequence of Lemmas 2.2 and 2.4. Since these sets are cones, with the null triple belonging to $\Pi_{1}^{4}$, only $\Pi_{1}^{4}$ could be closed. Once we prove that $\Pi_{1}^{1}$ is an open dense subset of $\Pi_{1}$, we must have int $\Pi_{1}^{j}=\emptyset, j=2,3,4$, and, by Theorem 3.1, none of the sets $\Pi_{1}^{j}, j=2,3,4$, can be open. Thus we just have to study $\Pi_{1}^{1}$ and $\Pi_{1}^{4}$.

Analyzing $\Pi_{1}^{1}$ : It is open because, by Lemma $2.4, \Pi_{1}^{1}=$ int $\Pi_{1}$, with this set being a dense subset of $\Pi_{1}$. Thus, $\Pi_{1}^{1}$ is a dense subset of $\Pi_{1}$.

Analyzing $\Pi_{1}^{4}$ : We have just to prove that $\Pi_{1}^{4}$ is non-closed. By Lemma 2.1, there exists $S \subset T$ and $\varphi \in \mathcal{C}(T)$ be such that $0 \in \operatorname{acc} \varphi(S) \subset \mathbb{R}_{++}$. Let $\left\{\mu_{r}\right\} \subset$ $\mathbb{R}_{++}$be an increasing sequence such that $\mu_{r} \rightarrow \frac{\pi}{2}$ and consider the sequence $\left\{\pi^{r}\right\} \subset \Pi$ such that $\pi^{r}:=\left(a^{r}, b^{r}, c^{r}\right)$, where $a^{r}=\left(\varphi \cos \mu_{r}+\varphi^{2} \sin \mu_{r}, 0_{n-1}\right)$, $b^{r}=\varphi \sin \mu_{r}-\varphi^{2} \cos \mu_{r}$ and $c^{r}=e_{1}, r=1,2, \ldots$. We have $c \in M_{r}$ and $\operatorname{int} M_{r}=\emptyset$. Moreover, $\left(a_{\bar{t}}^{r}, b_{\bar{t}}^{r}\right)=0_{n+1}$, so that Slater condition fails, and $\left(0_{n}, 1\right) \notin \operatorname{cl} K_{r}$. Hence $\left\{\pi^{r}\right\} \subset \Pi_{1}^{4}$ and $\pi^{r} \rightarrow \pi=(a, b, c)$, with $a=\left(\varphi^{2}, 0_{n-1}\right)$, $b=\varphi$ and $c=e_{1}$. Since $\left(0_{n}, 1\right) \in \operatorname{cl} K, c \in M$, and $\{c\} \times \mathbb{R} \subset K$, we get $\pi \in \Pi_{3}$. Hence $\Pi_{1}^{4}$ is non-closed.

In Example 2.3, the given neighborhood of $\pi$ is contained in $\Theta_{1}^{2}$ although the Slater condition holds for all its elements. Thus Theorem 3.3(ii) fails in general LSIP.

As a consequence of Theorem 3.3, the elements of the refined primal-dual partition are neither open nor closed, with the unique exception of $\Pi_{1}^{1}$, which is open. The density of $\Pi_{1}^{1}$ in $\Pi_{1}$ means that primal-dual solvability, with bounded optimal set, is a generic property in $\Pi_{1}=\Pi_{B}^{P} \cap \Pi_{B}^{D}$. The next corollary improves this generic result.

Corollary 3.4 Primal-dual solvability, with bounded optimal sets, is a generic property in the set $\Pi_{B}^{P} \cup \Pi_{B}^{D}$. 
Proof By Theorem 3.3(i), $\Pi_{1}^{1}$ is open and dense in $\Pi_{1}$. By Lemma 2.4(vii), $\Pi_{5} \cup \Pi_{6} \subset \operatorname{cl} \Pi_{1} \subset \operatorname{cl} \Pi_{1}^{1}$. Therefore $\Pi_{B}^{P} \cup \Pi_{B}^{D}=\Pi_{1} \cup \Pi_{5} \cup \Pi_{6} \subset \operatorname{cl} \Pi_{1}^{1}$.

Example 2.3 shows that the continuity assumption is essential for the validity of this generic result. In fact, the open neighborhood of $\pi$ is contained in $\Theta_{S}^{P}$ whereas all its elements are contained in $\Theta_{B}^{D} \backslash \Theta_{S}^{D}$, i.e., int $\Theta_{S}^{P}$ contains an open set of parameters which are not even dual solvable. Hence the primal-dual solvability is not a generic property in $\Theta_{B}^{P}$ (or in $\Theta_{B}^{D}$ ) and Corollary 3.4 is not true in general LSIP.

\section{The refined primal and dual partitions}

COROLLARY 4.1 The elements of the refined primal partition are neither open nor closed and satisfy the following statements:

(i) $\pi \in \Pi_{I C}^{P}$ if and only if $\left(0_{n}, 1\right) \in \mathrm{cl} K$. Moreover, int $\Pi_{I C}^{P}=\operatorname{int} \Pi_{3}$ is dense in $\Pi_{I C}^{P}$.

(ii) $\pi \in \Pi_{S}^{P}$ if and only if $\left(0_{n}, 1\right) \notin \mathrm{cl} K$ and $c \in \operatorname{int} M$. Moreover, $\operatorname{int} \Pi_{S}^{P}=\Pi_{1}^{1}$ is dense in $\Pi_{B}^{P}$.

(iii) $\pi \in \Pi_{N}^{P}$ if and only if $\left(0_{n}, 1\right) \notin \mathrm{cl} K$ and $c \notin \operatorname{int} M$, and $(\{c\} \times \mathbb{R}) \cap \mathrm{cl} K \neq$ $\emptyset$ if $c \in M$. Moreover, int $\Pi_{N}^{P}=\emptyset$.

(iv) $\pi \in \Pi_{U B}^{P}$ if and only if $\left(0_{n}, 1\right) \notin \mathrm{cl} K, c \notin M$ and $(\{c\} \times \mathbb{R}) \cap \operatorname{cl} K=\emptyset$. Moreover, int $\Pi_{U B}^{P}=\operatorname{int} \Pi_{2}$ is dense in $\Pi_{U B}^{P}$.

Proof (i) It is straightforward consequence of the existence theorem and [13, Theorem 2(i)] (it also follows from Lemma 2.4, parts (iii), (iv), (vi), (vii)).

(ii) The characterization of $\Pi_{S}^{P}$ was established in Lemma 2.2(i). This, together with Table 2 and Lemma 2.4(i) gives $\Pi_{1}^{1} \subset \Pi_{S}^{P} \subset \Pi_{1}$. On the other hand, by Lemma 2.4(i) and Theorem 3.3, int $\Pi_{1}=\Pi_{1}^{1}$. Hence int $\Pi_{S}^{P}=\Pi_{1}^{1}$. The density of $\Pi_{1}^{1}$ in $\Pi_{B}^{P}$ follows from the density of $\Pi_{1}^{1}$ in $\Pi_{1}$ (again by Lemma 2.4(i)) and the density of $\Pi_{1}$ in $\Pi_{1} \cup \Pi_{5}=\Pi_{B}^{P}$ (by Lemma 2.4(vii)).

(iii) The characterization of $\Pi_{N}^{P}$ is consequence of those corresponding to the remaining elements of the refined primal partition. Concerning int $\Pi_{N}^{P}$, observe that $\Pi_{N}^{P}=\Pi_{5} \cup \Pi_{1}^{3} \cup \Pi_{1}^{4}$. Since $\Pi_{5} \subset \operatorname{clint} \Pi_{1}$ and $\Pi_{1}^{3} \cup \Pi_{1}^{4} \subset \Pi_{1}$, we have $\Pi_{N}^{P} \subset \operatorname{clint} \Pi_{1}=\operatorname{cl} \Pi_{1}^{1}$, with $\Pi_{N}^{P} \cap \Pi_{1}^{1}=\emptyset$. Then $\operatorname{int} \Pi_{N}^{P}=\emptyset$.

(iv) It is Lemma 2.4(ii) because $\Pi_{U B}^{P}=\Pi_{2}$.

It remains to prove that the elements of the refined primal partition are neither open nor closed.

The elements of the refined primal partition are cones, and the null parameter belongs to $\Pi_{N}^{P}$, so that the remaining elements are non-closed. On the other hand, since there exists a sequence $\left\{\pi^{r}\right\} \subset \Pi_{1}^{4} \subset \Pi_{N}^{P}$ such that $\pi^{r} \rightarrow \pi \in \Pi_{3} \subset$ $\Pi_{I C}^{P}$ (recall the last part of the proof of Theorem 3.3), $\Pi_{N}^{P}$ is non-closed too.

On the other hand, since the null parameter belongs to $\Pi_{N}^{P} \backslash\left(\operatorname{int} \Pi_{N}^{P}\right), \Pi_{N}^{P}$ is 
non-open. Concerning $\Pi_{S}^{P}$, (ii) asserts that int $\Pi_{S}^{P}=\Pi_{1}^{1} \neq \Pi_{S}^{P}$ because $\Pi_{1}^{2} \neq \emptyset$ (Theorem 3.1), so that $\Pi_{S}^{P}$ cannot be open. Finally, $\Pi_{U B}^{P}=\Pi_{2}$ is non-open by Lemma 2.4(ii) and the same applies to $\Pi_{I C}^{P}$ by [13, Proposition 2].

The proof of the next result is similar to the last one and will be omitted.

COROLLARY 4.2 The elements of the refined dual partition are neither open nor closed and satisfy the following statements:

(i) $\pi \in \Pi_{I C}^{D}$ if and only if $c \notin M$. Moreover, int $\Pi_{I C}^{D}=\operatorname{int} \Pi_{2}$ is dense in $\Pi_{I C}^{D}$. (ii) $\pi \in \Pi_{S}^{D}$ if and only if Slater condition holds and $c \in M$. Moreover, int $\Pi_{S}^{D}=\Pi_{1}^{1}$ is dense in $\Pi_{B}^{D}$.

(iii) $\pi \in \Pi_{N}^{D}$ if and only if Slater condition fails and $c \notin M$, and $\{c\} \times \mathbb{R} \nsubseteq K$ if $\left(0_{n}, 1\right) \in \operatorname{cl} K$. Moreover, int $\Pi_{N}^{D}=\emptyset$.

(iv) $\pi \in \Pi_{U B}^{D}$ if and only if $\left(0_{n}, 1\right) \in \operatorname{cl} K, c \in M$ and $\{c\} \times \mathbb{R} \subset K$. Moreover, int $\Pi_{U B}^{D}=\operatorname{int} \Pi_{3}$ is dense in $\Pi_{U B}^{D}$.

Observe that, from Corollaries 4.1 and 4.2, part (ii), we get that $\Pi_{1}^{1}$ is dense in $\Pi_{B}^{P} \cup \Pi_{B}^{D}$. This is an alternative proof of Corollary 3.4.

Corollary 4.2(ii) fails in the context of general LSIP, as Example 2.3 shows. The situation is much better in the open and closed linear subspace of the parameters with bounded data, say $\Gamma$, which is formed by those triples $(a, b, c) \in \Theta$ such that the functions $a$ and $b$ are bounded. Obviously, if $T$ is a compact Hausdorff space, then $\Pi \subset \Gamma$. In [11] (using ad hoc tools for $\Gamma$ introduced in [15]) and in [16] it is proved that the strong uniqueness of the primal problem and the primal-dual solvability are generic properties in $\Gamma_{B}^{P}$ and in int $\Gamma_{B}^{P}$, respectively. The next example shows that the generic property proved in this paper fails in $\Gamma$.

Example 4.3 Let $T=[0,1]$ and $n=2$. Let $\pi=(a, b, c)$ be such that $a_{t}=$ $(t, 1)$ for all $t \in T$,

$$
b_{t}=\left\{\begin{array}{l}
1, \quad \text { if } t=0 \\
0, \quad \text { if } 0<t<1 \\
-1 \text { if } t=1,
\end{array}\right.
$$

and $c=\left(\frac{1}{3}, 1\right)$. Since $F=\left\{x \in \mathbb{R}^{2} \mid x_{1}+x_{2} \geq 0, x_{2} \geq 1\right\}, x^{*}=(-1,1)$ is strongly unique solution of $\pi$ and $\pi \in \Gamma_{S}^{P}$. Even more, since $c \in \operatorname{int} M$, we have $\pi \in \operatorname{int} \Gamma_{S}^{P}$. Nevertheless, since there is no duality gap (because $c \in \operatorname{int} M$ ) and $(\{c\} \times \mathbb{R}) \cap K$ is not closed, we have $\Lambda^{*}=\emptyset$ and, so, $\pi \in \Gamma_{B}^{D}$ but $\pi \notin \Gamma_{1}^{1}$. This means that $\operatorname{int} \Gamma_{S}^{P} \neq \Gamma_{1}^{1}$ (i.e., Theorem 4.2(ii) fails). Consider the sequence $\pi^{r}=\left(a^{r}, b^{r}, c^{r}\right), r=2, \ldots$, such that $a^{r}=a, c^{r}=c$ and $b_{t}^{r}=b_{t}$ if $t \neq \frac{r-1}{r}$ and $b_{t}^{r}=\frac{1}{r}$ if $t=\frac{r-1}{r}, r=1,2, \ldots$ Given $r \geq 2$, we have $F_{r}^{*}=\left\{x^{*}\right\}$ (because 
$\operatorname{cl} K_{r}=\operatorname{cl} K$ is equivalent to $\left.F_{r}=F\right)$ and $\Lambda_{r}^{*}=\left\{\lambda^{r}\right\}$, with

$$
\lambda_{t}^{r}=\left\{\begin{array}{cc}
\frac{2 r-3}{3(r-1)}, & \text { if } t=0, \\
\frac{r}{3(r-1)}, & \text { if } t=\frac{r-1}{r}, \\
0, & \text { otherwise. }
\end{array}\right.
$$

Since $\left\{\pi^{r}\right\} \subset \Gamma_{1}^{1}$ and $\pi^{r} \rightarrow \pi$, we have $\pi \in\left(\operatorname{cl} \Gamma_{1}^{1}\right) \backslash \Gamma_{1}^{1} \subset \operatorname{bd} \Gamma_{1}^{1}$ (as expected, taking into account the mentioned generic results).

Concerning the dual problem in continuous LSIP, examples have been given in [23] of compact Hausdorff spaces $T$ such that, in an open set, the dual problems have more than one optimal solutions, so that the generic results on unicity of saddle points fail. This implies that the problem of obtaining generic results for the dual problem is very difficult even in the continuous case. The extension of the generic results in this paper from continuous LSIP to LSIP with bounded data will be the object of further study.

Acknowledgement. The authors wish to thank one anonymous referee for its valuable comments and suggestions.

\section{References}

[1] Auslender, A., Teboulle, M., 2003, Asymptotic Cones and Functions in Optimization and Variational Inequalities (New York: Springer-Verlag)

[2] Ben-Israel, A., Charnes, A., Kortanek, K.O., 1969, Duality and asymptotic solvability over cones, Bull. Amer. Math. Soc. 75, 318-324

[3] Cánovas, M.J., López, M.A., Parra, J., Toledo, F.J., 2005, Distance to ill-posedness and consistency value of linear semi-infinite inequality systems, Math. Programming 103A, 95-126

[4] Cánovas, M.J., López, M.A., Parra, J. and Toledo, F.J., 2006, Ill-posedness with respect to the solvability in linear optimization. Linear Algebra Appl., 416, 520-540

[5] Cánovas, M.J., López, M.A., Parra, J. and Toledo, F.J., 2006, Distance to solvability/unsolvability in linear optimization, SIAM J. Optim., 16, 629-649

[6] Christov, G., Todorov, M.I., 1988, Semi-infinite optimization. Existence and uniqueness of the solution, Math. Balk. 2, 182-191

[7] Goberna, M.A., 2005, Linear semi-infinite optimization: recent advances. In: A. Rubinov and V Jeyakumar (Eds.) Continuous Optimization: Current Trends and Applications, 3-22 (New York: Springer-Verlag)

[8] Goberna, M.A., López, M.A., 1998, Linear Semi-Infinite Optimization (Chichester: John Wiley \& Sons)

[9] Goberna, M.A., López, M.A., Todorov, M.I., 1996, Stability theory for linear inequality systems, SIAM J. Matrix Anal. Appl. 17, 730-743

[10] Goberna, M.A., López, M.A., Todorov, M.I., 2001, On the stability of the feasible set in linear optimization, Set-Valued Analysis 9, 75-99

[11] Goberna, M.A., M.A. López, and M.I. Todorov, 2003, A generic result in linear semi-infinite optimization, Applied Mathematics and Optimization 48, 181-193

[12] Goberna, M.A., Todorov, M.I., Primal-dual stability in continuous linear optimization, Math. Programming (Series B), to appear

[13] Goberna, M.A., Todorov, M.I., Primal, dual and primal-dual partitions in continuous linear optimization, manuscript

[14] Greemberg, H.J., Pierskalla, W.P., 1975, Stability theory for infinitely constrained mathematical programs, J. Optim. Theory Appl. 16, 409-428 
[15] Helbig, S., Todorov, M.I., 1998, Unicity results for general linear semi-infinite optimization problems using a new concept of active constraints, Appl. Math. Optim. 38, 21-43

[16] Hernández, L., Todorov, M.I., 2005, Examples of bad phenomena of the dual problems and generic results in parametric linear semi-infinite optimization, Comt. Rend. Acad. Bulg. Sci. 58, $1247-1251$

[17] Kallina, C., Williams, A.C., 1971, Linear programming in reflexive spaces, SIAM Rev. 13, 350376

[18] Kortanek, K.O., Zhang, Q., 2001, Perfect duality in semi-infinite and semidefinite programming, Math. Programming 91A, 127-144

[19] Nunez, M.A., 2002, A characterization of ill-posed data instances for convex programming, Math. Programming (Ser. A), 91, 375-390

[20] Robinson, S.M., 1975, Stability theory for systems of inequalities. Part I: Linear systems, SIAM J. Numer. Anal. 12, 754-769

[21] Sturm, J.F., 2000, Theory and algorithms of semidefinite programming. High performance optimization, Appl. Optim. 33, 1-194

[22] Todorov, M.I., 1985-86, Generic existence and uniqueness of the solution set to linear semiinfinite optimization problems, Numer. Funct. Anal. Optim. 8, 541-556

[23] Todorov, M.I., 1989, Uniqueness of the saddle points for most of the Lagrange functions of the linear semi-infinite optimization, Numer. Funct. Anal. Optim. 10, 367-382 